\title{
Acute human cytomegalovirus infection among voluntary blood donors in the Lake Victoria zone blood transfusion centre: should it be considered in screening?
}

\author{
Erius Tebuka ${ }^{\dagger 1}$, Ruharara D Fulgence ${ }^{2}$, Bertrand Msemwa $^{3}$, Vitus Silago ${ }^{2}$, \\ Stephen E Mshana ${ }^{2}$, Mariam M Mirambo ${ }^{+2}$
}

1. Department of Pathology, Weill Bugando School of Medicine, Catholic University of Health and Allied Sciences, P.O. Box 1464, Mwanza, Tanzania

2. Department of Microbiology and Immunology, Weill Bugando School of Medicine, Catholic University of Health and Allied Sciences, P.O. Box 1464, Mwanza, Tanzania

3. Institute of Allied Health Sciences, Catholic University of health and allied sciences, P.O. Box 1464, Mwanza, Tanzania

Emails: ET: eriust@yahoo.com; FDL: fulgenceruharara@gmail.com; BM b.msemwa@yahoo.com; VS: vsilago.silago2@gmail.com; SEM: mshana72@yahoo.com; MMM: mmmirambo@gmail.com

\begin{abstract}
Background: Despite blood transfusion being a lifesaving option, it may be associated with blood borne infections including human cytomegalovirus(HCMV). The World Health Organization recommends screening of blood products for HCMV before transfusion to pregnant women, neonates and immunocompromised patients. However, this is not routinely practised in many resource limited countries.

Objective: This study aimed at determining seroprevalence of specific HCMV IgM antibodies among volunteered blood donors at the Lake Victoria zone blood transfusion centre

Methods: A total of 228 sera from volunteered blood donors were analyzed using HCMV $\operatorname{IgM} \mu$ capture enzyme linked immunosorbent assay as per manufacturer's instructions. Data were analyzed by STATA version 13

Results: The median age of the study participants was 19 interquartile range (IQR): 18-23 years. The seroprevalence of specific HCMV IgM antibodies was found to be 23/228 (10.1\%, 95\% confidence interval (CI): 6-14. None of the factors was found to be associated with HCMV IgM seropositivity among blood donors.

Conclusion: One out 10 blood donors in the Lake Victoria zone blood transfusion centre is acutely infected with HCMV. There is a need to consider screening of HCMV before blood transfusion particularly in resource limited countries where HCMV is endemic.
\end{abstract}

Keywords: Human cytomegalovirus, Tanzania, blood transfusion.

DOI: https://dx.doi.org/10.4314/ahs.v19i3.7

Cite as: Tebuka E, Fulgence RD, Msemwa B, Silago V, Mshana SE, Mirambo MM. Acute human cytomegalovirus infection among voluntary blood donors in the Lake Victoria zone blood transfusion centre: should it be considered in screening? Afri Health Sci. 2019;19(3): 2351-2355. https://dx.doi.org/10.4314/abs.v19i3.7

\section{Introduction}

Blood transfusion is a medical intervention with an es-

\section{Corresponding author:}

Mariam Mirambo,

Microbiology/Immunology department

P.O. Box 1464, Mwanza

Fax: +255282502678

Email: mmmirambo@gmail.com sential role in patient management particularly in emergency cases. Although it is lifesaving, it may be a potential risk for transmitting blood borne infections ${ }^{1}$. The World Health Organization (WHO) recommends that blood for transfusion should be screened for blood-borne infections such as Hepatitis C Virus, Hepatitis B Virus (HBV), Treponema pallidum, and Human Immunodeficiency Virus (HIV) on routine basis ${ }^{2}$. In addition, the WHO also recommends that blood to be transfused to neonates, preg-

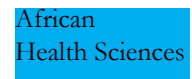

(C) 2019 Tebuka et al. Licensee African Health Sciences. This is an Open Access article distributed under the terms of the Creative commons Attribution License (https://creativecommons.org/licenses/BY/4.0), which permits unrestricted use, distribution, and reproduction in any medium, provided the original work is properly cited. 
nant women and immunocompromised patients should be screened for Human cytomegalovirus (HCMV) ${ }^{3}$. However, in most of resource limited countries HCMV is not considered in any of these cases. HCMV infection in infants is associated with meningitis, pneumonia and sepsis while infection in pregnant women may lead to congenital infections that may cause life threatening congenital anomalies. Furthermore, HCMV infection in immunocompromised individuals is associated with severe life threatening diseases such as meningitis and sepsis $^{4-76,8-11}$. It should be noted that, in immunocompetent individuals, acute primary HCMV infection is often self limited followed by latency mostly in $\mathrm{CD} 34^{+}$haemopoietic progenitor cell in bone marrow and $\mathrm{CD} 13^{+}, \mathrm{CD} 14^{+}$ peripheral blood mononuclear cells with potential of reactivation during immunocompromised state ${ }^{12}$.

Tanzania, like many other resource limited countries, the blood donors are not routinely screened for HCMV. Nonetheless, the donated blood has been also used for the management of pregnancy related anemia, immunocompromised patients as well as neonates in need. This poses a potential risk for transfused individuals which might be associated with life threatening clinical manifestations ${ }^{13,14}$. Despite HCMV being reported in a significant proportion of blood donors in many of the endemic countries $^{15-17}$, there is no routine HCMV screening with limited data among blood donors in Tanzania. This has prompted us to investigate the seroprevalence of acute/ recent HCMV infection among blood donors to provide appropriate recommendations for policy makers to consider it in screening particularly in case blood units are needed by vulnerable groups.

\section{Methods}

Study design, study area, sample size and ethical considerations

A cross-sectional study using 228 achieved sera collect- ed from blood donors aged 18 years collected between March 2016 and July 2017 was conducted in the city of Mwanza. The sample size was calculated using the Kish Leslie formula a prevalence of $12.2 \%$ from a previous study in Burkina-Faso was fitted in the formula ${ }^{18}$. The minimum sample size was 169 , however a total of 228 sera were retrieved and include into the study. Sera were conveniently selected until desired sample size was reached. All sera with incomplete information were excluded from the study. Socio-demographic and other relevant information were recorded from preexisting database. The protocol to conduct this study was approved by the joint CUHAS/BMC research ethics and review committee (CREC) with ethical clearance number CREC $340 / 2017$.

\section{Laboratory processing of the specimens}

Detection of specific HCMV IgM antibodies was done using commercial IgM $\mu$ capture enzyme linked immunosorbent assay (ELISA) according to manufacturer's instructions (PishtazTeb, Tehran, Iran). The assay has sensitivity and specificity of $>99 \% 0^{19-22}$.

\section{Data analysis}

Data was analyzed by using STATA version 13. Continous variables were summarized as median with interquartile range while categorical variables were summarized as proportions. Chi square test was used to test association between outcome and independent variables

\section{Results}

The median age of the study participants was 19(IQR:1823) years. Male sex was more predominant $84.65 \%$ $(193 / 228)$ compared to female sex. Most of the participants, $74.12 \%$ (169/228) were students (Table 1). 
Table 1: Characteristics of 288 blood donors attended at

Victoria zone blood transfusion center in Mwanza, Tanzania

\begin{tabular}{|c|c|c|}
\hline Participant characteristics & Number(Frequency) & Median (\%) \\
\hline Age & 228 & 19 (IQR:18-23) \\
\hline Female & 35 & $15.35 \%$ \\
\hline Male & 193 & $84.65 \%$ \\
\hline Occupation: Workers & 59 & $25.88 \%$ \\
\hline Students & 169 & $74.12 \%$ \\
\hline CMV IgM: Negative & 205 & $89.91 \%$ \\
\hline Positive & 23 & $10.09 \%$ \\
\hline
\end{tabular}

The overall seroprevalence of specific HCMV IgM antibodies was found to be $23 / 228(10.09 \%$, 95\% CI: 6-14). Out of 35 females, 2(5.7\%) were IgM seropositive compared to $21(10.9 \%)$ of 193 males, $\mathrm{P}=0.35$. Regarding occupation, $13.6 \%$ of 59 workers were seropositive compared to $8.9 \%$ of 169 students, $\mathrm{P}=0.304$. The seroprevalence in age (years) groups; ${ }^{16-25,26-45}$ and 36-45 were $10.1 \%, 12.9 \%$ and $9.1 \%$, respectively. On Wilcoxon ranksum (Man Whitney) test, there was no significant difference in age among positive cases and negative cases (19, IQR: $18-25$ vs. 19 , IQR: 18-22, P>0.05). Among socio-demographic characteristics assessed, none of them was found to be associated with HCMV IgM seropositivity among blood donors in the Lake Victoria zone blood transfusion center.

\section{Discussion}

This is the first study to establish the seroprevalence of specific HCMV IgM antibodies among blood donors in the Lake Victoria zone blood transfusion center. In the current study, a significant proportion of blood donors had specific IgM antibodies which often indicate acute or recent infections. Results from the current study are comparable to a previous report from Ouagadougou Burkina Faso which reported the prevalence of $12.2 \%{ }^{18}$. In comparison to other previous studies, the reported seroprevalence in this study is lower than $85 \%$ and $28 \%$ reported in North-West Iran and in Nigeria ${ }^{16,23}$. On the contrary, the reported seroprevalence in this study is indeed high compared to the previous reports in India and Kenya which documented the seroprevalence of $0.07 \%$ and $3.6 \%$, respectively ${ }^{15,24}$. The possible explanation for these variations could be geographical variations, climatic and socio-economic backgrounds which might influence the epidemiology of the HCMV ${ }^{25,26}$. Another explanation could be sensitivity and specificity of the test used in different studies. In the current study, IgM $\mu$ capture ELISA was used with sensitivity and specificity of $100 \%$ and 99\%, respectively which might be different from other studies. Considering the fact that HCMV is not routinely screened in most of resource limited countries where the prevalence is high, this might increase the risk of transmission to vulnerable groups such as immunocompromised patients, neonates and pregnant women. This underscores the need to consider screening for HCMV in donated blood before transfusion particularly in vulnerable groups.

In the current study, none of the socio-demographic factors assessed was found to be associated with HCMV IgM seropositivity. This could be explained by the fact that the current study used achieved sera which might limit accessibility of other information from the study participants. Further studies to explore more on the risk factors are recommended in this setting.

\section{Conclusion and recommendations}

A substantial proportion of blood donors in the Lake Victoria zone blood transfusion centre is acutely infected with HCMV which calls for the need to consider screening for HCMV before the donated blood is transfused to recipients especially vulnerable groups. Further studies to explore more on the risk factors are highly recommended in this setting. 


\section{Competing interests}

None declared

\section{Acknowledgements}

The authors would like to acknowledge the technical support provided by Mr. Seif Abdu, and all staff at the lake zone blood transfusion centre and the Bugando medical centre laboratory.

\section{Authors' contributions}

ET, MMM and SEM participated in the design of the study. MMM, BM and RDF participated in the collection of specimens and clinical data. RDF performed serological tests. SEM and ET analysed and interpreted the data. MMM wrote the first draft of the manuscript. SEM and ET did critical review of the manuscript. All authors read and approved the final version of the manuscript.

\section{References}

1. Cheesbrough M: District laboratory practice in tropical countries: Cambridge University Press; 2006.

2. Epstein JS, Holmberg JA: Progress in monitoring blood safety. Transfusion 2010, 50(7):1408-1412.

3. Organization WH: Screening donated blood for transfusion-transmissible infections: recommendations: World Health Organization; 2010.

4. Jeena PM, Coovadia HM, Chrystal V: Pneumocystis carinii and cytomegalovirus infections in severely ill, HIV-infected African infants. Annals of Tropical Paediatrics 1996, 16(4):361-368.

5. Kovacs A, Schluchter M, Easley K, Demmler G, Shearer W, Russa PL, Pitt J, Cooper E, Goldfarb J, Hodes D: Cytomegalovirus infection and HIV-1 disease progression in infants born to HIV-1-infected women. New England Journal of Medicine 1999, 341(2):77-84.

6. Mwaanza N, Chilukutu L, Tembo J, Kabwe M, Musonda K, Kapasa M, Chabala C, Sinyangwe S, Mwaba P, Zumla A: High rates of congenital cytomegalovirus infection linked with maternal HIV infection among neonatal admissions at a large referral center in sub-Saharan Africa. Clinical Infectious Diseases 2013, 58(5):728-735.

7. Adler SP, Chandrika T, Lawrence L, Baggett J: Cytomegalovirus infections in neonates acquired by blood transfusions. Pediatric Infectious Disease 1982, 2(2):114-118. 8. Mirambo MM, Chibwe E, Mushi MF, Majigo M, Mshana SE: Cytomegalovirus, Parvovirus B19 and Rubella
Co-infection among Pregnant Women Attending Antenatal Clinics in Mwanza City: The Need to be considered in Tanzanian Antenatal Care Package. Epidemiology: Open Access 2016, 2016.

9. Bello C, Whittle H: Cytomegalovirus infection in Gambian mothers and their babies. Journal of Clinical Pathology 1991, 44(5):366-369.

10. Chibwe E, Mirambo MM, Kihunrwa A, Mshana SE: Magnitude of the Cytomegalovirus infection among pregnant women attending antenatal clinics in the city of Mwanza, Tanzania. BMC Research Notes 2017, 10(1):489.

11. Mirambo MM, Senyaeli N, Mshana SE: Low humoral responses to human cytomegalovirus is associated with immunological treatment failure among HIV infected patients on highly active antiretroviral therapy. Pan African Medical Journal 2017, 28(131).

12. Roback JD: CMV and blood transfusions. Reviews in Medical Virology 2002, 12(4):211-219.

13. Champoux JJ, Drew W, Neidhardt F, Plorde JJ: Sherris medical microbiology. In.: New York: The McGraw-Hill Companies, Inc; 2004.

14. Griffiths P, Baraniak I, Reeves M: The pathogenesis of human cytomegalovirus. The Journal of Pathology 2015, 235(2):288-297.

15. Njeru D, Mwanda W, Kitonyi G, Njagi E: Prevalence of cytomegalovirus antibodies in blood donars at the National Blood Transfusion Centre, Nairobi. East African Medical Journal 2009, 86(12):58-61.

16. Eivazi-Ziaei J, Movassagpour A, Asgharzadeh M, Dastgiri S: Seroprevalence of cytomegalovirus in blood donors in the northwest of Iran. Journal of Analytical Research in Clinical Medicine 2013, 1(2):96-100.

17. Mahmood R, Malik F, Hussain S, Ashfaq K, Akhtar T, Alam M: Seroprevalence of cytomegalovirus among blood donors in local population. Int J Pharm Chem 2014, 4:146-150.

18. Ouedraogo A, Yameogo J, Poda G, Kientega Y, Ouédraogo TR: Prevalence of anti-CMV antibodies in blood donors in Ouagadougou (Burkina Faso). Medecine et sante tropicales 2012, 22(1):107-109.

19. Souza VAUFd, Sumita LM, Otsubo MES, Takei K, Pannuti CS: Enzyme linked immunosorbent assay for rubella antibodies: a simple method of antigen production. A preliminary report. Revista do Instituto de Medicina Tropical de São Paulo 1995, 37(4):357-359.

20. Field P, Ho D, Cunningham A: Evaluation of rubella 
immune status by three commercial enzyme-linked immunosorbent assays. Journal of Clinical Microbiology 1988, 26(5):990-994.

21. Chernesky M, Wyman L, Mahony J, Castriciano S, Unger J, Safford J, Metzel P: Clinical evaluation of the sensitivity and specificity of a commercially available enzyme immunoassay for detection of rubella virus-specific immunoglobulin M. Journal of Clinical Microbiology 1984, 20(3):400-404.

22. Schwarz TF, Jäger G, Gilch S: Comparison of seven commercial tests for the detection of Parvovirus B19-specific IgM. Zentralblatt für Bakteriologie 1997, 285(4):525-530. 23. Oladipo E, Akinpelu O, Oladipo A, Edowhorhu G: Seroprevalence of Cytomegalovirus (CMV) among
Blood Donors at Bowen University Teaching Hospital Ogbomoso. American Journal of Medical and Biological Research 2014, 2(3):72-75.

24. Kumar H, Gupta P, Kumar S, Sarkar R: Is seroprevalence of anti-IGM CMV among blood donors relevant in India? Indian Journal of Pathology and Microbiology 2008, 51(3):351.

25. Stagno S, Cloud GA: Changes in the epidemiology of cytomegalovirus. In: Immunobiology and Prophylaxis of Human Herpesvirus Infections. Springer, 1990: 93-104. 26. Zhao P, Ma D, Xue F, Ji C, Wang S, Zhang X, Zhou $\mathrm{Y}, \mathrm{Yu}$ X: Seroprevalence and risk factors of human cytomegalovirus infection in the Eastern Chinese population. Archives of virology 2009, 154(4):561-564. 